\title{
A COMBINED STUDY OF ART WORKS PRESERVED IN THE ARCHAEOLOGICAL MUSEUMS: 3D SURVEY, SPECTROSCOPIC APPROACH AND AUGMENTED REALITY
}

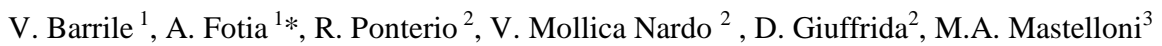 \\ ${ }^{1}$ Mediterranea University - DICEAM- Department of Civil Engineering - Via Graziella Feo Di Vito Reggio Calabria Italy - \\ (vincenzo.barrile, antonino.fotia)@unirc.it \\ ${ }^{2}$ IPCF-CNR of Messina Italy - ponterio@ipcf.cnr.it \\ ${ }^{3}$ Director of Polo, Archeologic Park and Museum "L. Bernabò Brea" of Lipari Italy;
}

KEY WORDS: 3D model, Spectroscopy, Virtual Reality

\begin{abstract}
:
The renewed interest, in the context of international museological studies, for historical installations such as museographic devices (able to give shape to a space duly dialoguing with the user), is due today to their communicative immediacy. In recent years the availability of libraries and tools for the use of augmented reality (AR) content has undergone an important increase. AR innovation represents a new method for enhancing the presence visitors into the museum industry despite its use leads to an increase in costs and instrumental investments. In this regard, in a wider work of valorisation and dissemination of archaeological heritage, we are working on the development of an app for tourism purposes. The aim of this paper is the application of some techniques (3D modelling, spectroscopy, virtual reality) to study, record and make available information about archaeological artefacts preserved in the Regional Museum of Lipari (clay masks) and in the National Archaeological Museum of Reggio Calabria (the statue Kouros and the Amphoras). The three-dimensional models produced are well suited to applications of augmented reality for the promotion and dissemination of information on the archaeological /architectural/cultural heritage.

Moreover, the integration of the information about characterization of materials are essential in order to identify both geographic area of provenience and manufacturing historic period. Some pigments or medium, and in general all materials, can be indeed connected to a determinate historic time or to a specific artist; to this particular purpose spectroscopic methods are valid approach for their non-destructive nature.
\end{abstract}

\section{INTRODUCTION}

Characterization of materials used in works of art is essential in order to identify both geographic area of provenience and manufacturing historic period. Some pigments or medium, and in general all materials, can be indeed connected to a determinate historic time or to a specific artist; to this particular purpose spectroscopic methods are valid approach for their nondestructive nature. Moreover, since the traditional graphic documentation for Cultural Heritage will be destined to be replaced by three-dimensional digital models, the creation of these detailed representations will be deployed as valid tools for monitoring of conservation status, of recomposition and restoration activities, of virtual fruition etc.

The three-dimensional models produced are well suited to applications of augmented reality for the promotion and dissemination of information on the archaeological/ architectural/cultural heritage.

The aim of this paper is the application of some techniques (3D modelling, spectroscopy, virtual reality) to study, record and make available information about archaeological artefacts preserved in the Regional Museum of Lipari (clay masks) and in the National Archaeological Museum of Reggio Calabria (the statue Kouros and the Amphoras).

A combination of non-invasive spectroscopic approach and 3D documentation by laser scanning system using portable instrumentations was carried out. These methods are nondestructive and they don't require preparation of samples.
In this paper the artefacts have been surveyed using both a Faro laser arm (model Cam2 Arm Quantum) and a photographic acquisitions (Canon Eos 7D) using a 'converging axes' schema, in order to get models not only geometrically accurate but also having a photorealistic rendering.

Moreover the renewed interest, in the context of international museological studies, for historical installations such as museographic devices (able to give shape to a space duly dialoguing with the user), is due today to their communicative immediacy. Additionally, in recent years the availability of libraries and tools for the use of augmented reality content has undergone an important increase. AR innovation represents a new method for enhancing visitors into the museum industry despite concerns over its return on investment. In this regard, in a wider work of valorisation and dissemination of archaeological heritage, we are working on the development of an educational app for tourism purposes.

\section{CASI STUDIO}

\subsection{Kouros}

The statue of Kouros, stylistically it dates to around $500 \mathrm{BC}$, is among the major representations of the archaic sculpture of Calabria and exhibited today in the Archaeological Museum of Reggio Calabria. It was probably sculpted by a local artist working in Rhegion (ancient Reggio Calabria) or Eastern Sicily. It is very simple in its forms and is made in white marnle brought in from the Greek island of Paros in the Cyclades.

\footnotetext{
* Corresponding author
} 
The methods and the exact location of the discovery are not known for now, it is reasonably supposed in the building excavations carried out on the waterfront of the city.

The statuette of the Kouros of Reggio Calabria was found fortunately, during a tax police inspection at the home of a wealthy local businessman.

The statue represents a young man between 18 and 20 years of age in the fullness of his physical and spiritual beauty, with a face animated by lips that seem to pose a sort of archaic smile, a thick hair with a refined hairstyle composed of four rows of curls around the forehead, long vertical curls and knotted braids on the nape (Fig.1).

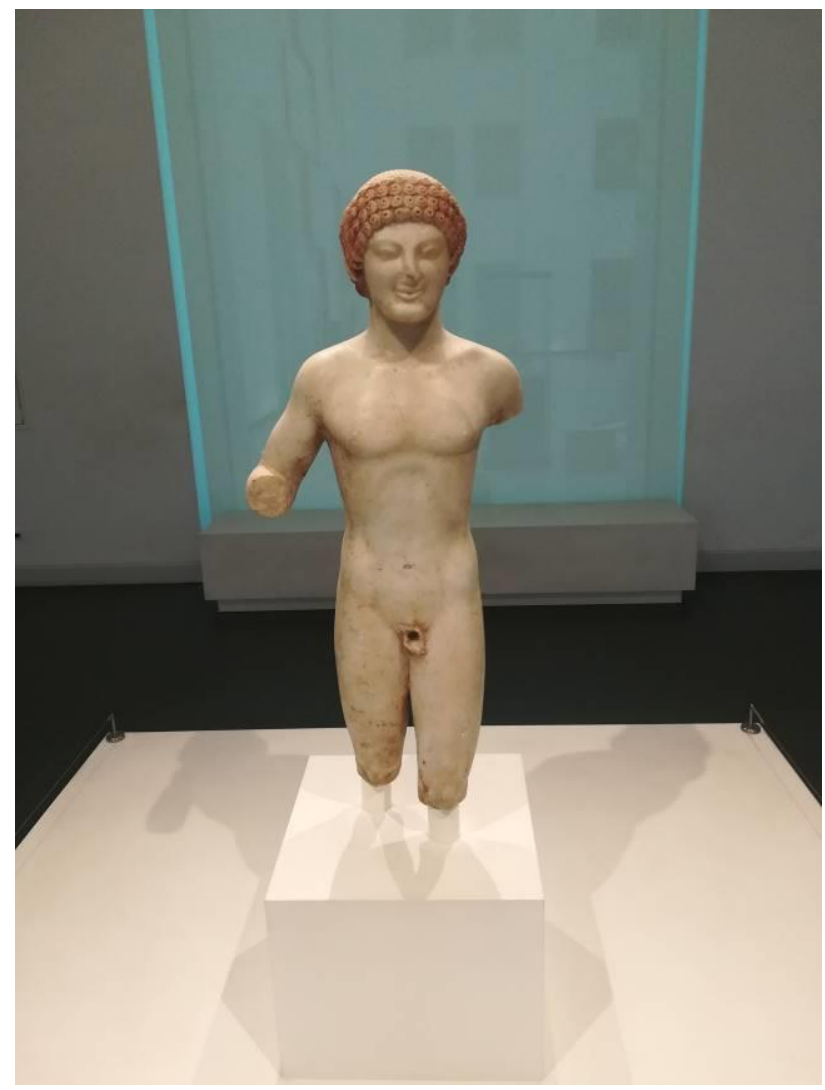

Figure 1. Kouros

The arms, only partially preserved, were probably extended in a gesture of offering to the divinity. In fact, this type of statuette was widely used in the archaic period as a votive object, very often found in the burial necropolis of the deceased, or in sanctuaries, as a gift to the venerated divinity.

The Kourioi, plural of Kouros, in the culture of ancient Greece, symbolized the masculine aesthetic perfection, to which corresponded an absence of emotional expressions that betrayed the inner and spiritual peace, for this they were objects suitable for burials, which accompanied the deceased with excellent credentials to the gods.

At that time in the ancient colony of Rhegion there was an aristocratic government that had brought prosperity and prosperity. It is not excluded that among the many well-to-do of Rhegion, someone has commissioned the statuette more as a votive offering than as a funerary outfit since there are no traces of necropolis.

\subsection{Anfore Dressel}

To reconstruct what were the navigation and trade routes along the Calabrian coasts in Roman times, important indicators are African ceramics, fine tableware that replaces Italic and Gallic productions, conquering the Mediterranean markets between the 2nd and 3rd century AD.

Another important testimony is offered by the logs of anchors in lead and by the amphorae of various origins and chronology exhibited in the Archaeological Museum of Reggio Calabria. The Dressel amphora (the name refers to Heinrich Dressel, 1845-1920, who was one of the first to study and classify the amphorae and stamps on the amphorae themselves) is the most common amphora of the late Roman Republican age (Fig.2).

Begins to be produced between 140/130 BC and persists until the middle of the first century BC.

Its diffusion in the second and first half of the I century BC constitutes the most important evidence of the export of the agricultural production of Italy in the ancient world.

A large number of these amphorae have been found mainly in the western Mediterranean basin, while a smaller number in the eastern basin.

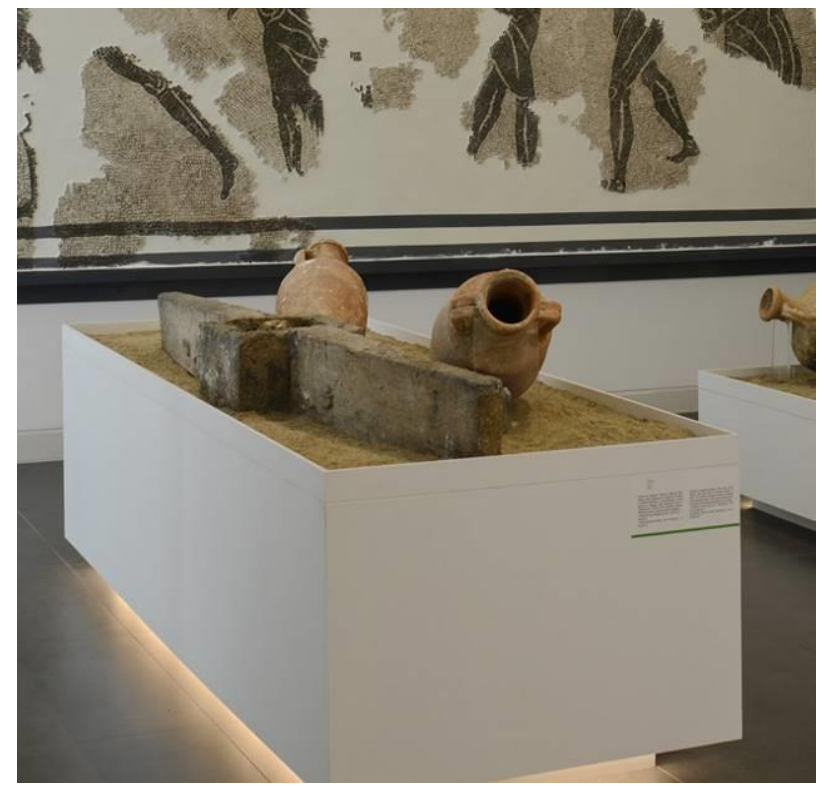

Figure 2. Dressel Amphorae

Both the terrestrial and marine finds are testimony to this. In fact, this type of amphora has been found in large quantities in various wrecks. For example, many wrecks have been found along the coasts of Gaul (present-day France) and near the coast of the Iberian peninsula.

A large number of Dressel amphorae found throughout the Mediterranean have the name of Marco Sestio associated with the anchor. He was a member of an important senatorial family and owner of large wine estates.

\subsection{Clay Mask}

An extraordinary collection of masks and statuettes of a theatrical subject found in the grave goods of the fourth and third centuries $\mathrm{BC}$ and preserved in the classical section of the Aeolian Museum of Lipari, they document a little-known cult of Dionysus present in the funerary art of Magna Graecia. These 
are more than a thousand pieces of theatrical terracottas (4th and 3rd centuries), that constitute the richest, most complete and ancient documentation relating to the stage costume of the Greek age.

The fact that these terracottas have been found in funerary objects reveals the close connection between the cult of the dead and the Dionysian. Dionysus is in fact the god of wine, drunkenness and ecstasy that brings joy to convivial gatherings and is also the god of theatre who originated in Greece at parties in his honour.

But Dionysus is also the god who allowed his followers to tap into the eternal beatitudes of the afterlife. The close connection between the theater and funerary Dionysism is present in this period throughout the Magna Graecia and in Sicily and takes on a particularly rich and documented aspect in Lipari.

In fact, the small masks discovered inside the tombs are also not isolated but have been found in groups of two or more (up to eight) and certainly in non-random associations: they correspond to the characters of the tragedies of Sophocles and Euripides or of the comedies of Aristophanes. Some of them have in common an extraordinary expressiveness and characterization that suggests that it is not a production of local craftsmanship, but rather a reproduction of Athenian prototypes inspired in turn by the original masks created by the tragedians themselves on the occasion of the presentation of their works in competitions in Athens (Fig.3).

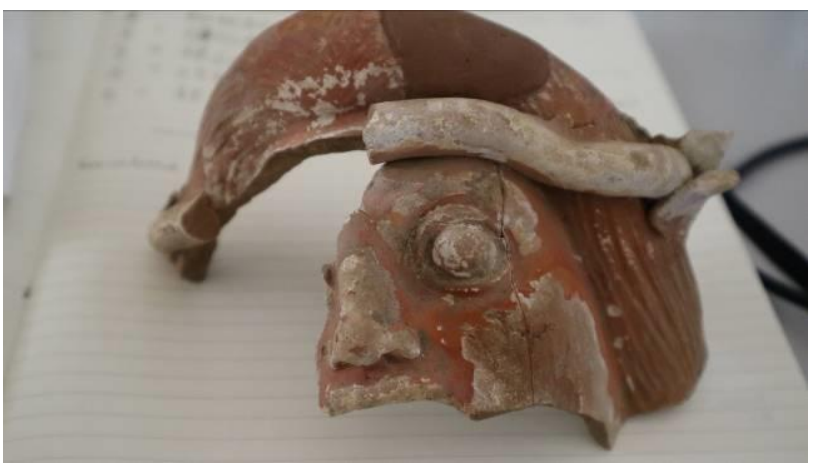

Figure 3. Dressel Amphorae

\section{3D MODELS}

\subsection{Data acquisition}

Three-dimensional reconstruction using photogrammetry and laser scanner are widely used in different fields, from architecture engineering, medical sector, to archaeology and cultural heritages.

For the acquisition of digital images useful for the construction of a geometric 3D model was used a Sony Alpha 7 III (Sony, Japan) mounted on a tripod. The Sony Alpha 7 III is a full frame digital camera with CMOS Exmor R sensor (35,6 × 23,8 mm), 24,2 megapixel with FE 24-105 mm F4 G OSS objective. The scans instead were carried out using a Faro laser arm (model Cam2 Arm Quantum). This laser scanner has a high rate of sample capture $(19,200$ point and normal samples per minute) with very high precision (35 microns between samples). The scanner arm is secured to ensure the motion of the arm does not warp the scanning surface. Each scan was completed in two parts, one of the obverse side and one of the reverse side.

The scans of each side were overlapped to facilitate alignment between the scans.

\subsection{Data Processing}

The acquired photograms were processed using the Agisoft Photoscan software. The workflow is completely automatic both as regards the orientation of the images and for the generation and reconstruction of the model. This condition led to an optimization of processing times ensuring good performance of the machine/software complex (Balletti et al, 2015) (Canciani et al 2003).

The phases of the elaboration were the following:

1. Align photos (photo alignment); This is the most important phase of the entire photogrammetric process. In this phase, the software aligns our photographs with each other, calculating their position in space and reconstructing the so-called gripping geometry. Then, through a process of geometric triangulation, calculate the position in space of the elements present in the photographs. It follows that the quality of this alignment derives the goodness of the final 3D model.

The result of this phase is a sparse point cloud.

Following the alignment, measurement of the control points takes place, to register the block in real world coordinates, in the reference system defined by the control points.

2. Build Dense Cloud; Through this phase, a dense cloud is constructed using dense image matching algorithms. These are subdivided into algorithms that use a stereo pair to find matches and those that identify them in multiple images;

3. Extraction mesh; it consists in generating a polygonal model based on the newly created dense cloud. The mesh is a subdivision of a solid into smaller solids of a polyhedral shape; 4. Build texture; it instead allows obtaining the $3 \mathrm{D}$ representation of the work under investigation.

The last step is the scaling of the model, that is the assignment of the correct metric dimension to the model, in order to make precision measurements on it (Bonora V. and Tucci G. 2008). Following this scheme the models of the works preserved in the Museums have been reproduced (Fig.4,5)

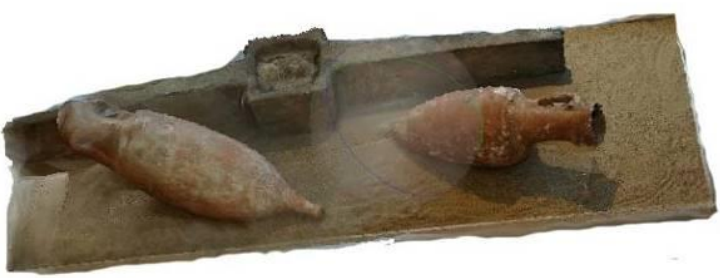

Figure 4. 3D Model Dressel Amphorae

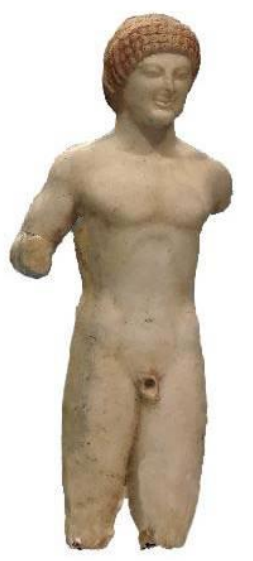


Figure 5. 3D Model Kouros

In relation to the laser scanner data processing the resolution of the obtained scans was set to a $0.2 \mathrm{~mm}$ grid, with a close interval between lines $(1.0 \mathrm{~mm}$ spacing); the obtained "raw data" had to be reworked.

Each scan was completed in two parts, one of the obverse side and one of the reverse side.

The scans of each side were overlapped to facilitate alignment between the scans. Once both sides were scanned, both point cloud sets were edited to remove the surface of the table on which the objects were set during the scanning process, as well as any other extraneous data that was acquired.

The point cloud for each side of the object was then aligned to the other and combined to make a single point cloud that represented all sides of each artifact.

Once the point clouds were complete, they were converted to meshes and exported as .stl files to be aligned to the meshes created in Photoscan.

Once both the photogrammetric model and the laser scanned model are imported into Geomagic Wrap.

So, once aligned, the photogrammetric mesh is replaced by the laser scanned mesh and the texture map is re-mapped to the laser-scanned mesh (Barazzetti L, Remondino F, Scaioni M, 2010).

The result is a 3D model with the photographic accuracy of photogrammetic 3D models and the geometric precision of laser scanned 3D models (Fig.6,7) (Micheletti, N., Chandler, J.H. and Lane, S.N. 2015). Measurements taken from visual cues of the photographic texture are more accurate due to the increased fidelity of the underlying mesh, and the incidence of inadequate model detail being mistaken for an actual characteristic of an artifact instead of an erroneous model should be reduced (Green, S., Bevan, A. and Shapland, M. 2014).

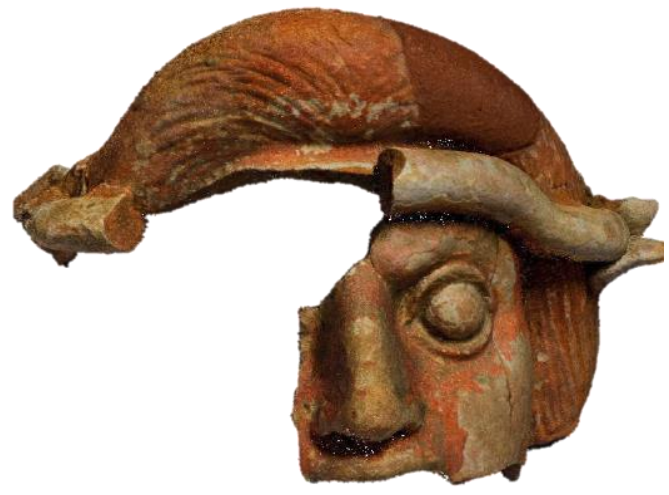

Figure 6. Point cloud

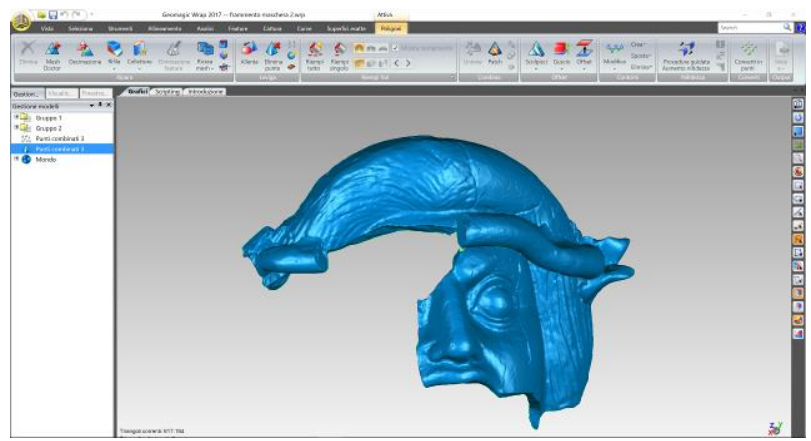

Figure 7. 3D Model Clay Mask

\section{SPECTROSCOPY}

Raman spectroscopy is a non-destructive, superficial technique (it analyzes layers a few microns thick, less than the diameter of a hair), based on the interaction between radiation and material. Raman spectroscopy is based on the Raman effect, and the inelastic scattering of a monochromatic radiation interacting with the sample is measured.

Generally, a light beam that affects a sample passes through it without being altered or it is absorbed depending on the wavelength of the radiation and the nature of the under study material. There are also phenomena of diffusion: the "scattering" that a beam of electromagnetic wave undergoes crossing a medium because of the interactions with the particles. A small part of the incident beam is spread elastically, ie with the same frequency: this is the Rayleigh effect. The radiation produced by the Raman diffusion is recorded and a graph of wavelengths (based on the intensity) is elaborated below. This spectrum can be associated with a single molecule and constitutes a real digital imprint (Burgio L. and Clark R.H. 2001).

The applications are numerous: the characterization of pigments, dyes and binders to that of ceramic surfaces, stone materials, organic substances of various nature, the identification of degradation products on pictorial surfaces, glass, ceramics, metals and rocks, etc..

The types of information that can be obtained with this technique can be summarized in the following points:

- Study of "intramolecular" modes (molecular properties) and intermolecular modes (properties of the crystal lattice);

- Measurements in polarized light to investigate molecular order, anisotropy, alignment and molecular self-organization and crystalline domains;

- Mapping on the surfaces of the samples to monitor the spatial distribution of structural inhomogeneities (chemical, physical, phasic, etc.) with a spatial resolution of the order of one $\mu \mathrm{m}$. From the point of view of the sampling depth, the analysis carried out with a Raman spectrometer is an analysis of a superficial type: the information comes from a layer a few $\mu \mathrm{m}$ thick on the surface. For this reason, it is easy to understand that the most useful applications of Raman spectrometry interest the characterizing of the surface properties of a sample, eg. degradation products, pigments on a painting or a manuscript, etc.

From the point of view of cultural heritage the application is therefore important as well as the identification of the historical period of belonging and the geographical location of origin of the works of art investigated also in relation to their biodegradation. In fact on the works colonies of microorganisms often develop in favorable conditions whose metabolic activity leads to the creation of crusts that alter chromaticity (the presence of calcium monohydrate and carotenoids, a symptom of biological activity on the part of lichens).

\subsection{Raman spectroscopy analysis}

In order to the Raman effect occur, the sample must be irradiated with visible or near-infrared wavelength radiation, but due to the small Raman emission fraction (1/109 photons reemitted), the use of an intense light source, that is the motivation because a monochromatic, coherent, collimated and focused laser source is used. Furthermore, to minimize any interference due to Rayleigh diffusion, that being more intense than the Raman lines would tend to cover the signal, a high quality optics is required (Conti C., et all, 2016). 
In the specific, the clay masks have been analysed with Bruker BRAVO Handheld Raman Spectrometer equipped with licensed Duo Laser system and algorithm to mitigate the fluorescence, providing reliable and quality spectra on both inorganic and organic substances (SSE TM, Sequentially Shifted Excitation). Raman spectroscopy is able to characterize the pigment contained in masks a non-destructive way. The Raman spectra are acquired in 300-3000 cm-1 spectral range with an exposure no longer than $20 \mathrm{~s}$. The spectra are processed with OPUS software and the identification of the collected signals by comparison with a spectral database of reference materials (Fig. 8) (Mollica Nardo V., et all. 2017).

The Raman spectrometry technique is a molecular type technique that allows information to be obtained in terms of functional groups. The identification of the analyzed substances is carried out by comparison with standard reference spectra. Today there are many online bookstores (www.irug.org) (www.ehu.eus/udps/database/database.html) that allow us to compare the results obtained.

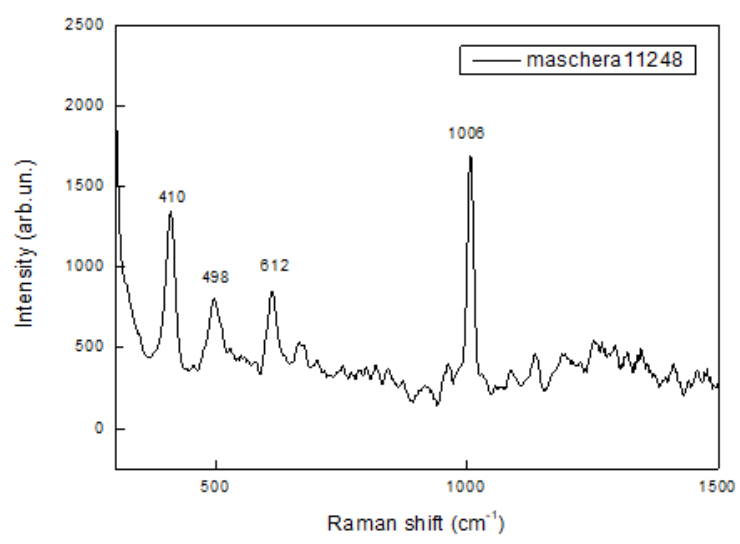

Figure 8. The raman spectrum shows bands at 410, 498 and 612 $\mathrm{cm}-1$ attributable to hematite pigment and a band at $1006 \mathrm{~cm}-1$ identifiable with gypsum. Raman spectra are acquired on red pigment in three different points of clay mask fragment $\mathrm{n}^{\circ}$

11248.

\section{THE DEVELOPED APP}

\subsection{Integration of 3D models in Virtual Reality}

The use of 3D, in this field, is aimed at acquiring a knowledge of Cultural Heritage otherwise precluded to the mass or otherwise difficult to access without being in an invasive approach to an extremely vast, ancient and in some cases delicate heritage. (Cuca, et all. 2011)

The only physical contact of a mold with artwork could damage the statues, leave marks or abrasions that could jeopardize the works. With 3D scanning we can see how it is possible to obtain a highly detailed model of the statue without any physical contact with it (Rinaudo F, Bornaz L, Ardissone P, 2007), and can then recreate an almost identical copy of the original .

Even more evident is the way in which modern technology can help to make information attractive proposing it in different and often playful ways; i.e. an interactive activity exploited in museums to realize guided paths of the works or to make available the vast knowledge that does not it can be materially contained in one building.

Over the last few decades the museum has abandoned its simple container structure, becoming a livelier and richer reality and a communication tool that aims to exploit modern technologies to attract the public.

Another important application of three-dimensional models is represented by the integration of the $3 \mathrm{~d}$ models in environments of augmented reality.

In fact, today museums play an important role in society. They support the task of collecting, preserving, researching and presenting objects and materials from different historical periods. The responsibility of the museum is to take the events of the past and show them in contemporary contexts with the aim of bringing information and entertainment to the public.

Although many museums still tend to have a conservative style to present their exhibits, a large number of the latter understand that "modern times call modern measures" and therefore make continuous efforts to explore different ways to interact with their audience. And right now this means incorporating experiences into augmented reality.

The augmented reality allows us to see a level of digital content in a real-life scenario; for this reason many museums are embracing the ability to give visitors a richer view of history. The improvement of ths methodologies allows museums to shows how history can be brought back to life with the use of augmented reality.

A Virtual reality system is generated by the combination of the real scene seen by the user and the pre-registered digital virtual scene, which can be significantly influenced by the behaviour of the active subject. The subject can "assist" a representation in a real space, or he can "explore" the real environment to which, in any case, virtual elements are superimposed.

Augmented Reality is an effective tool to promote the process of disseminating information and enhancing cultural heritage. It is a technology that allows adding virtual content to a physical environment interactively and in real time.

This technology, in addition to enriching virtual contents with what is real, makes it possible to activate the connections that man perceives in the world around him.

The enhancement of information extends the limits of knowledge because, thanks to the connection of every physical element to a telecommunications network, it creates a space in which everything is reachable through devices capable of accessing the Net, characterized by ever-greater portability. Labels and tags allow to visualize and share knowledge through links that are no longer verbal and therefore no longer confined between linguistic communities, but visual and therefore understandable by anyone, opening the doors to a global sharing of knowledge that sees the virtual level converge towards the current one.

The new technology allows to add 3D virtual content to a physical environment, in an interactive and real-time format. Unlike what happens with Virtual Reality, the Augmented Reality does not replace physical reality. It overlaps computer data to the real environment so that the user can have the feeling of being physically present in the landscape that sees. He reread on the screen and consequently the reality perceived by the subject is "augmented" by virtual objects that provide additional information on the real environment.

The founding elements of any Augmented Reality system are tracking, real-time rendering and visualization technologies.

In particular, tracking is fundamental. It is about registering the position of the observer, of real and fictitious objects. Precise and fast tracking is essential for the correct visualization of the virtual components of the scene. Information on observer position data can also be acquired through low-cost solutions such as cameras, which may be based on whether predefined objects are used as landmarks in the scene. 


\subsection{Unity and Vuforia}

Unity is a multiplatform tool for creating interactive 3D content, such as architectural visualizations or 3D animations in real time, using two different programming languages, customized JavaScript based on languages. NET and C ++.

The Unity development environment runs on both Microsoft Windows and MacOS, and the produced apps can also run on Android mobile devices.

From an operational point of view, Unity's programming work is based on the use of objects: the so-called "Game Objects".

To these elements, which may or may not have a graphical representation, scripts can be associated, which allow to define their behavior using particular event functions.

A fundamental part of any Augmented Reality system is the integration between real images and virtual information. This process, based on artificial vision techniques, has as its purpose the unification of the two reference systems taken into consideration: the virtual one and the real one.

Vuforia is the most used platform in the world for the development of augmented reality. Thanks to compatibility with the major mobile phones, tablets and mobile devices, there are more than 30,000 applications that use Vuforia loaded on the AppStore and Google Play.

Vuforia provides simple solutions for complex problems in the development of virtual reality.

From an operational point of view, this SDK is based on realtime tracking of images and objects. On these targets or "Markers", it is possible to place virtual elements (3D models for example), which are then displayed on screen according to the observer's perspective. One of the main strengths is the freedom in choosing these Markers. In fact, the framework allows the recognition of words, objects and images, even more than one at the same time, previously scanned through special tools, made available to developers.

\subsection{The app created}

The app created is a tourist app for mobile device that allows the user in real time, as well as having additional information on the object of the investigation, even to be able to view the 3D model in AR. He so makes a virtual tour inside (with a viewer or simply through the screen of the device), as well as being "accompanied" during the visit by a virtual guide that interacts with the surrounding environment. (Bae, Golparvar-Fard, White, 2013).

The app allows to visualize the visitor's position inside the museum's floor plans, the names of the exhibition rooms, of the present artefacts associating their multimedia contents; it allows you to highlight details directly on the work, making it easier to understand the work, and also allows you to recall a virtual guide (video) that explains the story of the work itself, or reconstruct, in virtual environment, a work of art that it is broken (Fig, 9,10) (Barrile, et all, 2019).

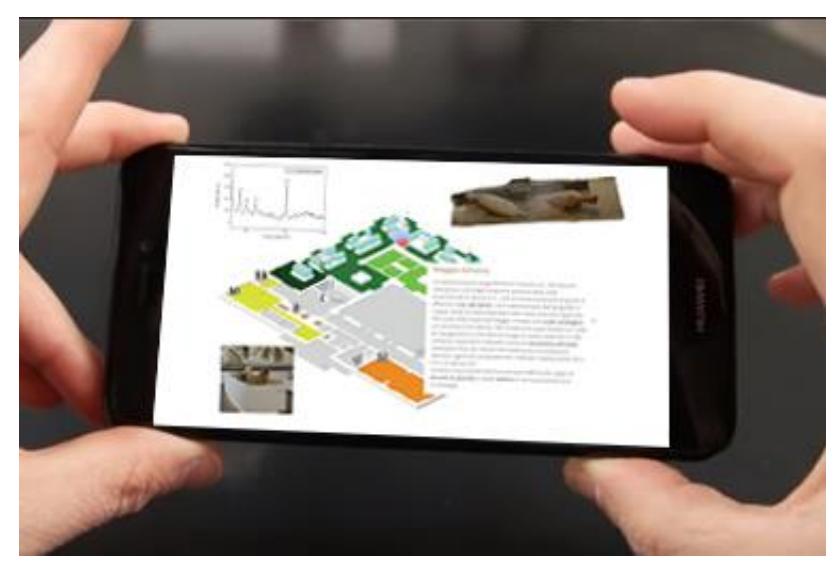

Figure 9. visitor's position inside the museum's floor plans, information regarding the art of work, 3D model and corresponding spectroscopy analysis.

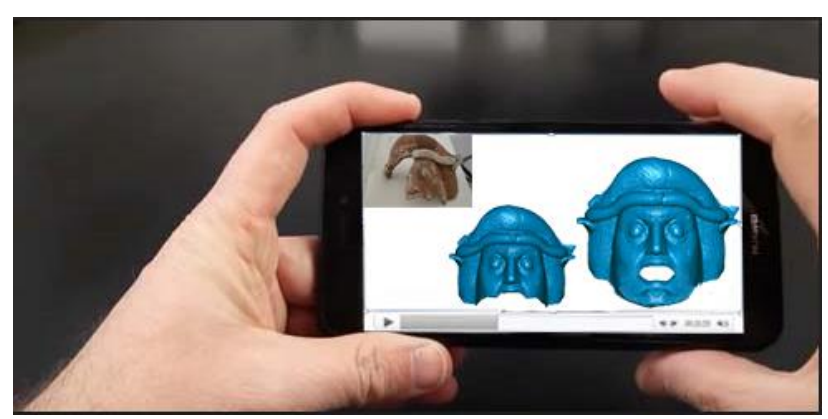

Figure 10. Mask reconstruction in VR

The programming work inside Unity is based on the use of objects: the so-called "Game Objects".

These elements, which may or may not have a graphic representation, can be associated with scripts, all extensions of the "Mono-Behavior" base class.

This Unity script, linked to a Game Object running on both the server and the client, is responsible for verifying in each frame the presence of new data inside the Buffer and therefore, based on these, to update its representation both on the server side that client side.

This effect is achieved thanks to the use of the RPC functions.

Therefore, based on the content of the buffer, the action to be taken on the object under examination is chosen and executed both on the version of the object maintained on the server and on those maintained on the clients. (Barrile, Fotia, Bilotta, 2018)

\section{CONCLUSION}

Today we are experiencing a revaluation of cultural heritage, no longer seen as the exclusive domain of specialized scholars, but as a resource for the economic development of local communities and regions.

In this process, the role of new technologies is fundamental: not only they help scholars to simplify the management and analysis of scientific data, but also they allow to better understand the past thanks also to interactive applications, personalized presentations and environments virtual realms.

The acquisition and 3D modeling systems have amply demonstrated that they can find fertile ground in the management and enhancement of Cultural Heritage. 
As known, the laser-scanner method is the most accurate and precise, futhermore thanks to the structure from motion algorithm (SfM), allows having a geometrical accuracy and effective photorealistic rendering. The two methods, however, prove to be complementary: it is possible to integrate the dense cloud from laser-scanner with the photographic texture taken from the cameras to finally get an integrated model.

The scanning and 3D modelling technologies have become in recent years a powerful tool for presenting and analyzing artwork in virtual places, such as the web, and in real places, such as museums or cities

All the examples reported on the use of technologies in the field of cultural heritage want to show the overcoming of the dichotomy tradition / innovation; we try to open the way to the sharing of the latter with new technologies through AR.

In this reality, the viewer is not limited to observing but also uses other senses to live a real experience to remember: thanks to digital 3D models and new technologies that allow us to explore them in an interactive way, the "passive" viewer becomes "active" user deciding what to see and the routes to do, sharing their opinions and feelings. In this way the experience will remain in visitor's memory.

Moreover, this kind of methodologies are useful to the scholars to share their research about the inspected work of art. A very useful example can be represented by the result of Raman Spectroscopy, infact, the identification and characterization of organic pigments used in works of art and cultural heritage play a fundamental role in order to detect not only a precise geographic area and a specific historic moment but also monitoring the work' $s$ art "health".

In this paper we work with Raman spectroscopy, largely used for the analysis of dyes of interest in the cultural heritage field, but unfortunately, sometimes pigments and dyes commonly employed in artistic production are often extremely fluorescent, making this detection methodology difficult or even unreliable. For this reason we would improve our work through the Surface Enhanced Raman Spectroscopy (SERS) that on the contrary satisfies many of the requirements of an ideal analytical technique in detecting chemical species in artworks.

\section{REFERENCES}

Bae H, Golparvar-Fard M, White J (2013) High-precision vision-based mobile augmented reality system for context-aware architectural, engineering, construction and facility management (AEC/FM) applications. Visual. Eng. 1(1):1-13

Barrile V., Fotia A., Bilotta G., Geomatics and augmented reality experiments for the cultural heritage in Applied Geomatics Vol. 10, Issue 4, pp. 569-578 (2018)

Barrile, V., Fotia, A., Bilotta, G., \& De Carlo, D. 2019 Jan 28. Integration of geomatics methodologies and creation of a cultural heritage app using augmented reality. Virtual Archaeology Review. [Online] 10:20

Barazzetti L, Remondino F, Scaioni M, 2010 Orientation and 3D modelling from markerless terrestrial images: Combining accuracy with automation. Photogrammetric Record 25(132): 356-381

Bonora V. and Tucci G. 2008, Strumenti e metodi di rilievo integrato, in Peroni A., Tucci G. (a cura di), Nuove Ricerche su Sant'Antimo, Firenze, Alinea.
Burgio L. and Clark R.H. 2001, Library of FT-Raman spectra of pigments, minerals, pigment media and varnishes, and supplement to existing library of Raman spectra of pigments with visible excitation, Spectrochim. Acta A 57.71491-521.

Chandler, J.H. and Lane, S.N., Structure from motion (SFM) photogrammetry. Geomorphological Techniques, 2015, 20470371 .

Conti C., Botteon A., Bertasa M., Colombo C., Realinia M. and Sali D., 2016 Portable Sequentially Shifted Excitation Raman spectroscopy as an innovative tool for in situ chemical interrogation of painted surfaces, Analyst 141.15 pp 4599-4607.

Cuca B, Brumana R, Scaioni M, Oreni D (2011) Spatial data management of temporal map series for cultural and environmental heritage. International Journal of Spatial Data Infrastructures Research, IJSDIR 6:1-31

English Heritage, 3D Laser Scanning for Heritage, English Heritage Publishing (2011).

English Heritage, Photogrammetric Applications for Cultural Heritage, English Heritage Publishing (2017).

Green, S., Bevan, A. and Shapland, M. 2014, A comparative assessment of structure from motion methods for archaeological research. Journal of Archaeological Science, 46, pp.173-181.

Micheletti, N., Chandler, J.H. and Lane, S.N. 2015, Structure from motion (SFM) photogrammetry. Geomorphological Techniques, , 2047-0371.

Mollica Nardo V., Aliotta F., Mastelloni M. A., Ponterio R. C, Saija F., Trusso S. and Vasi C. S. 2017, A spectroscopic approach to the study of organic pigments in the field of cultural heritage', Accademia Peloritana dei pericolanti, 95.

Rinaudo F, Bornaz L, Ardissone P, 2007 3D high accuracy survey and modelling for Cultural Heritage Documentation and Restoration. Vast 2007-future technologies to empower heritage professionals, November 26-29, 2007, Brighton, UK, Archaeolingua Hun, pp. 19-23

\section{ACKNOWLEDGEMENTS (OPTIONAL)}

The authors thank Dr. C. Melacrino, director of National Archaeological Museum of Reggio Calabria (Italy), and Dr. M.A. Mastelloni, Director of Polo, Archeologic Park and Museum "L. Bernabò Brea" of Lipari (Italy) for the cooperation during field tests. 